\title{
Turismo de bienestar. Nuevas oportunidades en la hotelería
}

\author{
Wellness tourism. New opportunities in the hotel industry
}

\author{
María Alejandra Rojas Rieckhof*
}

Escuela Profesional de Turismo y Hotelería,

Universidad de San Martín de Porres, Perú

\section{Resumen}

La actividad turística es un fenómeno social que evoluciona y se adapta a las tendencias. Cada vez más personas viajan para experimentar sensaciones, conectarse con el mundo, la naturaleza y conocer diversas sociedades y costumbres. Incluso, en medio de la pandemia por COVID-19, existe la necesidad de encontrar espacios -cuando se den las condiciones- para alejarse de lo cotidiano y aliviar el estrés. Mediante los viajes, se logra esta conexión entre naturaleza, cultura y yo interior, por lo que es necesario contar con productos turísticos especializados en turismo de bienestar.

Con el objetivo de demostrar la conexión entre el turismo y la salud, en este estudio, se presentan conceptos y teorías analizando temas de turismo de salud y bienestar. Además, se describen las últimas tendencias registradas de viajeros, hoteles saludables, así como tendencias digital detox.

Palabras clave: salud, turismo de salud, bienestar o wellness, digital detox.

\section{Abstract}

The tourist activity is a social phenomenon that evolves and adapts to trends. More and more people travel to experience sensations, to connect with

Este es un artículo Open Access bajo la licencia Creative Commons Atribución-NoComercial-Compartirlgual 4.0

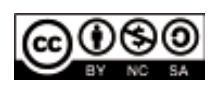


the world and nature, and to know diverse societies and customs. Even in the midst of the COVID-19 pandemic, there is a need to find spaces when the conditions are met to get away of everyday life and relieve stress. Through travel, this connection is achieved between nature, culture and the inner self, so it is necessary to have tourist products specialized in wellness tourism.

In order to demonstrate the connection between tourism and health, this study presents concepts and theories by analyzing issues of health and wellness tourism. In addition, the latest trends of travelers, healthy hotels, as well as digital detox trends, are described.

Keywords: health, health tourism, wellness, digital detox.

\section{Introducción}

Las prácticas turísticas en la postmodernidad implican buscar nuevas formas de expresión, por lo que las personas viajan para experimentar sensaciones, conectarse con el mundo, la naturaleza y conocer diversas sociedades y costumbres (Alberca, 2019). Estas manifestaciones culturales son en sí un potencial atractivo turístico, que unido a la naturaleza y técnicas ancestrales de salud y bienestar, son elementos cada vez más apreciados por los turistas. Mediante los viajes se logra esta conexión entre la naturaleza, la cultura y el yo interior, evadiendo de alguna forma, las labores cotidianas que suelen generar estrés.

Las prácticas de terapias de bienestar, que tienen como fin recuperar la salud emocional y física de los individuos, son un patrimonio inmaterial que las sociedades poseen; por lo general, son transmitidas de generación a generación y representan la autenticidad sociocultural de las comunidades que se construyen por medio de lo que se denomina memoria colectiva. Los colectivos pueden ser familias o pueblos que recuerdan hechos trascendentes, que guardan para sí mismos y que quedan como herencia común y que se denomina patrimonio del grupo (Urbano, 2000). 
El disfrute de la naturaleza es también parte del turismo cultural, puesto que la riqueza del medioambiente y paisaje forman parte de un patrimonio colectivo, donde se hace necesario centros de interpretación, que deben brindar información no solo del entorno natural, sino también de actividades de patrimonio cultural como la medicina alternativa o tradicional, terapias holísticas, entre otras (Paunero, 2004).

Con el objetivo de demostrar la conexión entre turismo y salud, en este estudio, se presentan conceptos y teorías analizando temas de turismo de salud y bienestar. Además, tomando en cuenta la pandemia por COVID-19 y sus implicancias en el turismo a nivel mundial, se describen las últimas tendencias registradas de viajeros, hoteles saludables, así como tendencias digital detox.

\section{Turismo de salud}

De acuerdo a lo señalado por Boullon, el hombre siempre ha buscado el equilibrio entre la salud y la vida cotidiana, aprovechando el beneficio del tiempo libre y las actividades de relax (como se citó en Rojas, 2018). La cultura griega y la cultura romana, en esto, pusieron especial énfasis al crear grandes estructuras de baños termales, algunas tan impresionantes como las Termas de Caracalla (Italia). La costumbre de buscar la salud o bienestar por medio de visitas a centros termales se extiende por todo Europa, como lo afirma Urry (2004); él describe, en sus investigaciones, el primer spa en Inglaterra que data de 1626; cuenta que médicos de la época recomendaban su uso. Algunos baños bastante reconocidos y visitados son los de Buxton, Harrogate, Tunbridge Wells y Badén Badén.

Cabe mencionar que en los centros termales se buscaba recobrar la salud con técnicas no invasivas, haciendo uso de elementos como el agua o métodos de relajación, prácticas de medicina alternativa y sabiduría ancestral. Lo que se sigue haciendo en la actualidad como parte del llamado turismo de bienestar o wellness.

El turismo de bienestar forma parte del turismo de salud, entendiéndose la salud como el estado completo de bienestar físico, mental y social y no solo como la ausencia de afecciones o enfermedad (Organización Mundial de la Salud [OMS], 2019). 
La motivación principal de viaje del turista es lo que define el tipo de turismo que realiza. De modo que, el turismo de salud, está referido al viaje que realiza una persona fuera de su residencia habitual para recibir un servicio de salud (Escuela de Organización Industrial [EOI], 2013, p. 10). Por su parte, Escalada y Romero (2015), consideran que turismo de salud es el viaje que realiza una persona a un destino para recibir tratamiento o terapia y recuperar la salud con descanso en dicho lugar. Es decir, se requiere para la operatividad del viaje, no solo un centro médico, hospital o clínica, sino una serie de instalaciones adicionales donde el turista descanse, se alimente y se relaje; todo ello, en busca de la recuperación del estado de bienestar ansiado.

La Organización Mundial de Turismo (OMT, 2019) describe al turismo de salud:

Aquellos tipos de turismo que tienen como motivación primordial la contribución a la salud física, mental y/o espiritual gracias a actividades médicas y de bienestar que incrementan la capacidad de las personas para satisfacer sus propias necesidades y funcionar mejor como individuos en su entorno y en la sociedad. El término turismo de salud engloba el turismo de bienestar y el turismo médico ...

El turismo médico es un tipo de actividad turística que implica la utilización de recursos y servicios de curación médica (tanto invasivos como no invasivos) con base empírica. Puede incluir el diagnóstico, el tratamiento, la cura, la prevención y la rehabilitación. (pp. 39, 41)

Por tanto, el turismo de bienestar es un tipo de actividad turística que aspira a mejorar y equilibrar los ámbitos principales de la vida humana, entre ellos el físico, mental, emocional, ocupacional, intelectual y espiritual. La motivación primordial del turista de bienestar es participar en actividades preventivas, proactivas y de mejora del estilo de vida, como la gimnasia, la alimentación saludable, la relajación, el cuidado personal y los tratamientos curativos.

Precisando, el turismo médico se subdivide en medicina curativa, medicina preventiva y medicina estética; mientras el turismo de bienestar es aquel que 
ayuda a mejorar las relaciones sociales e incrementa el grado de positivismo en las personas por medio de tratamientos alternativos de índole espiritual y mental, mejora la actividad física y ayuda a una mejor conexión con la naturaleza (MINCETUR, 2012).

El turismo de bienestar también es considerado como un subsegmento del turismo médico, específicamente de prevención de dolencias (EOI, 2013). Pero, la definición más utilizada para turismo de bienestar es la que lo considera una rama del turismo de salud que incluye tratamientos de relajación, tratamientos homeopáticos, en conexión con la medicina natural o relacionados al contacto con la naturaleza para recobrar la salud, como el termalismo o la talasoterapia (Arias et al., 2012, p. 76). Difiere del turismo médico que corresponde a atención médica, odontológica y quirúrgica.

El turismo médico es reactivo, responde a una necesidad de curación, el viaje se hace a un lugar fuera de la residencia habitual para tener un diagnóstico, tratamiento médico, operación, cura o rehabilitación de una enfermedad o patología (Deloitte, 2018). Mientras que el turismo de bienestar es proactivo, pues responde a una práctica de viaje que se hace para realizar un tratamiento terapéutico, preventivo de mejora de la salud física y mental, a la vez que se visita el destino y se realiza actividades turísticas en las que también se emplea equipamiento turístico (Tabla 1).

\section{Tabla 1}

Principales diferencias entre turismo médico y turismo de bienestar, wellness

\begin{tabular}{cll}
\hline \multirow{2}{*}{$\begin{array}{c}\text { ¿Qué buscan los } \\
\text { turistas? }\end{array}$} & \multicolumn{1}{c}{ Turismo de salud } & \multicolumn{1}{c}{ Turismo de bienestar } \\
\cline { 2 - 3 } & $\begin{array}{l}\text { Tratamientos médicos, } \\
\text { quirúrgicos, odontológicos, } \\
\text { diálisis, entre otros similares }\end{array}$ & $\begin{array}{l}\text { Tratamientos terapéuticos, } \\
\text { holísticos y medicina alternativa y/ } \\
\text { o tradicional }\end{array}$ \\
$\begin{array}{c}\text { ¿Dónde se puede } \\
\text { practicar? }\end{array}$ & $\begin{array}{l}\text { Clínicas y centros } \\
\text { médicos hospitalarios }\end{array}$ & $\begin{array}{l}\text { Hoteles, centros termales, } \\
\text { albergues, spas, lugares de retiro }\end{array}$ \\
$\begin{array}{c}\text { ¿Qué actividades se } \\
\text { realizan? }\end{array}$ & Procedimientos médicos & $\begin{array}{l}\text { Terapias alternativas, de } \\
\text { relajación, termalismo, } \\
\text { homeopáticas, o de conexión con } \\
\text { la medicina natural y tradicional }\end{array}$ \\
\hline
\end{tabular}

Nota. Elaboración propia. 
A pesar de que ambos, turismo médico y turismo de bienestar, tienen en el fondo la misma motivación de viaje: deseo de mejorar la salud; difieren en los productos utilizados para dicho fin. En el turismo médico, los productos son clínicas, hospitales, consultorios dentales, spas de terapias médicas, entre otros que guardan relación; mientras que en el turismo de bienestar los productos son hoteles spas, centros termales, lugares de retiro, cruceros de bienestar y centros ecológicos.

En la Figura 1 se muestra la variedad de productos usados en ambos tipos de turismo de salud, y los que están íntimamente ligados a un territorio, como los centros termales o lugares con terapias ayurvédicas o de sabiduría ancestral inca.

\section{Figura 1}

Productos de turismo de salud

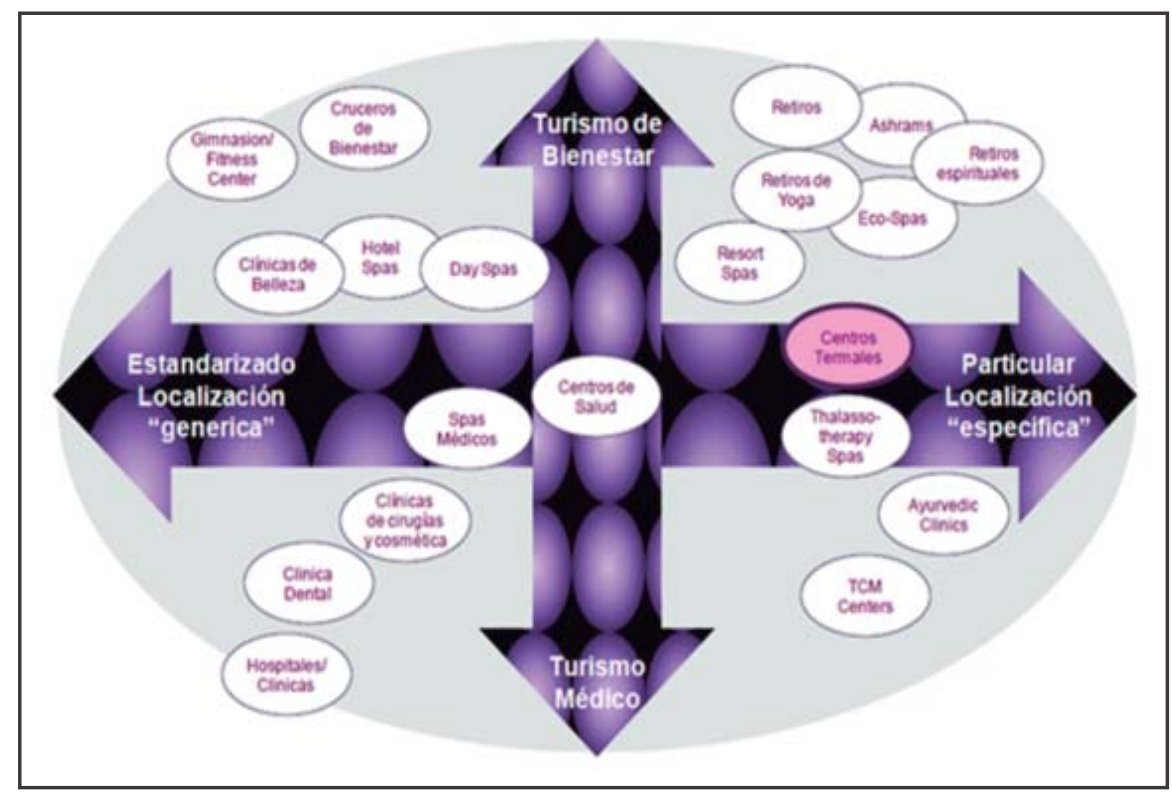

Nota. Global Spa Summit, Wellness Tourism and Medical Tourism: Where do Spas Fit Mayo 2011 (como se citó en MINCETUR, 2012). 


\section{Tendencias en turismo de bienestar}

En 2017, la OMT (2018) registró que 27\% de los turistas internacionales refirieron como motivo principal de viaje la salud, visita a parientes y/o amigos y religión; no se detalla qué porcentaje corresponde a turismo de bienestar o wellness. La inversión que hacen los turistas en actividades de turismo de salud lo hace un segmento muy atractivo (PROMPERU, 2018). En 2015 se registra a Estados Unidos como el principal destino a nivel mundial con un ingreso de \$3598 millones; en Latinoamérica los principales destinos registrados son Costa Rica (\$365 millones), México (\$280 millones), Panamá (\$40 millones) y Brasil (\$32 millones).

En cuanto a turismo de bienestar, es China el primer receptor de este segmento de mercado, seguido de Estados Unidos y Alemania; en Latinoamérica, países como México está en séptimo lugar y Brasil en octavo lugar, son los más demandados para este tipo de turismo; resaltando que el crecimiento anual del 2013 al 2015, en Brasil, es de 21\%; bastante mayor que México que es de $13 \%$.

Se considera como segundo factor importante en el crecimiento de la demanda por turismo de salud, al gasto que las personas hacen en ocio, con relación al relax y prevención de enfermedades, cuidado de la belleza y juventud. Lo ven como una buena inversión en asuntos vinculados al bienestar, por lo que buscan viajes saludables y escapadas de la rutina; logrando que estas actividades sean más solicitadas y se coticen mejor.

Existe una gran oportunidad para la creación de productos relacionados con el turismo de bienestar, dado su crecimiento a nivel mundial; estos productos de medicina holística ayurvédica, así como yoga, reiki, meditación, spa terapéuticos, centros de desintoxicación digital, etc. se pueden incluir en ofertas hoteleras tradicionales, esperando atraer al público de este segmento.

Las ciudades cada vez más urbanizadas y los trastornos relacionados al estrés conllevan a las personas a necesitar servicios especializados en estos temas. Al respecto, Ostelea (2018) menciona que el segmento de viajeros de bienestar crece de forma continua desde 2013 en adelante, $37.3 \%$ como se aprecia en la Figura 2. 
Figura 2

Tamaño del mercado del turismo de bienestar

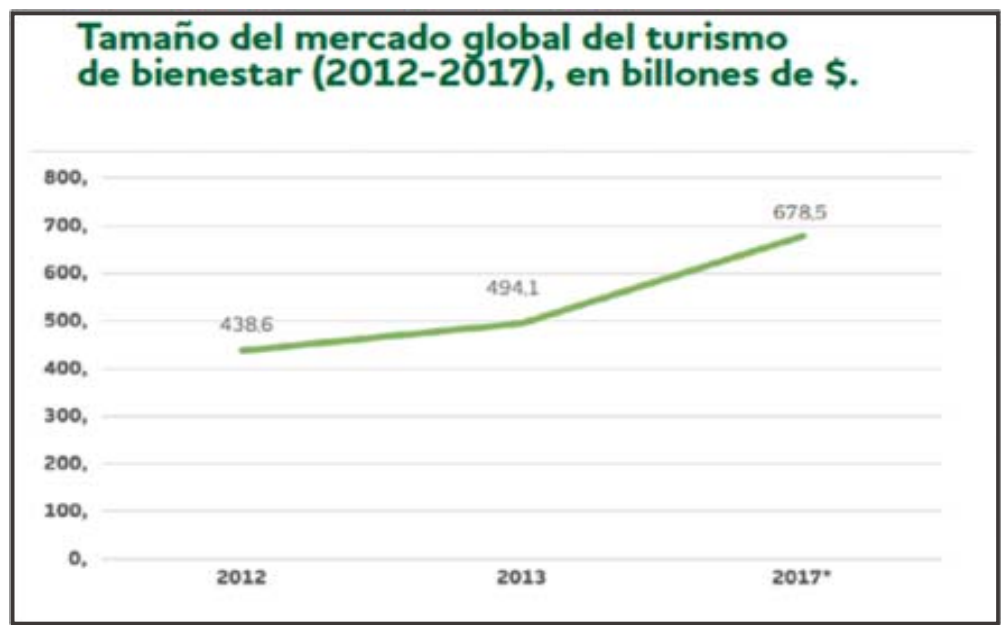

Nota. Global Spa Summit, s.f. Global market size of the wellness tourism industry from 2012 to 2017. En Statista - The Statistics Portal (como se citó en Ostelea, 2018).

En el Perú, el vacacionista nacional que busca hacer actividades relacionadas con la naturaleza llega a 56\%, de ese porcentaje 10\% visita lugares con aguas termales, sulfurosas o medicinales; acciones que están íntimamente ligadas al turismo de bienestar (PROMPERÚ, 2019).

La tendencia actual se relaciona con acciones de cuidado de la salud, cuidado personal y bienestar espiritual, donde la infraestructura turística es tipo termal, hoteles con spa, centros de talasoterapia y otros. Se vislumbra en este nuevo nicho de mercado creciente una buena oportunidad de negocio, al que pueden sumarse tratamientos estéticos, servicio nutricional, técnicas de meditación y relajación, y técnicas de enriquecimiento espiritual.

Los servicios más valorados por los turistas de bienestar son las terapias de medicina alternativa y tradicional, programas nutricionales para pérdida de peso, programas de ayuda para desórdenes del sueño, tratamiento de medicina ayurvédica, meditación y programas détox digital, estos últimos como una tendencia reciente, dirigido a personas que presentan dependencia de los medios digitales o enfermedades de adicción y estrés (Ostelea, 2018). 
De acuerdo a lo planteado, para elegir un destino, el turista de bienestar toma en cuenta tres factores clave:

1. La oferta de ocio con la que cuenta el destino. No solo busca tratamientos o actividades que mejoren su salud física y mental, sino que busca mediante la oferta de ocio conectarse con la cultura, naturaleza y gastronomía del destino, así como escaparse de la rutina y aprender de nuevas culturas.

2. Los costos de la oferta de turismo de bienestar. Se refiere a los costos en alojamiento, transporte y actividades complementarias y no solo a la terapia en sí.

3. La imagen del destino. Se desea vivir una experiencia auténtica y coherente con el bienestar, lo que implica seguridad, buena infraestructura turística, relajación, entorno natural y paisajístico, conocimiento ancestral, entre otros.

\section{Alojamientos saludables}

Los hospedajes saludables son una tendencia creciente a nivel mundial, lo comprueba las nuevas inversiones hoteleras en las que se incluyen spas, centros de tratamiento de salud y servicios relacionados con la salud y la belleza; que refuerzan su oferta con productos cada vez más especializados como comida vegana, programas antiestrés, técnicas de relajación y mindfulness. Como ejemplo está el grupo hotelero InterContinental que, bajo la marca EVEN en New York, está centrado en el bienestar, y el MGM Grand en las Vegas con un diseño de habitación saludable, que cuenta con duchas con infusiones de vitamina $\mathrm{C}$ y terapias para despertarse con luz y aromaterapia (SpaFinder Wellness, como se citó en EOI, 2013).

Existen hospedajes con un amplio repertorio de actividades medical wellness como el Villa Padierna Medical Wellness \& Spa en Málaga, España. También están los resorts del Club Med \& Beauty, que invitan a gozar de unas vacaciones saludables con oferta de relajación, como fitness y relax, yoga y pilates, sauna y hamman, entre otros productos para la salud y belleza (EOI, 2013). 
El Lima, se encuentra el Westin Lima Hotel \& Convention Center, con su oferta del producto Heavenly Spa By Westin, brindan el spa urbano como se ve en su página web; se señala que es el más grande de Sudamérica con diversos servicios de wellness como shiatsu, aspire massage, drenaje linfático y técnicas de masajes con piedras, plumas, entre otros.

Así pues, tanto la salud y el bienestar están íntimamente vinculados con el rubro turístico y hotelero, y el rubro médico. Empresas de salud que invierten en el sector hotelero como el grupo San Pablo que, como se indica en su página web, inició operaciones en 1991 y cuenta con tres unidades de negocio: salud, educación y hotelería, esta última cuenta con una red de hoteles entre los que se mencionan Aranwa Hotels Resorts \& Spas en Cusco, Valle Sagrado, Colca, Paracas y Vichayito; con característica de hospedajes de temática wellness, donde se ofrecen diferentes tipos de terapias alternativas y programas wellness que complementan la salud física y enriquecen la salud espiritual.

\section{Digital detox nueva tendencia en el mundo wellness}

El término digital detox se concibe como el período de tiempo en el cual una persona se abstiene del uso de la tecnología -ya sea teléfonos inteligentes o computadoras- con el fin de reducir el estrés y poder enfocarse en la interacción social y el entorno (Oxford English Dictionary, 2021). Situación cada vez más urgente en una sociedad altamente supeditada a la tecnología, dadas las adicciones tecnológicas que van en desmedro de la salud.

Escalada y Romero (2015), refieren que esta tendencia comenzó en la industria turística con una campaña dentro de los restaurantes quienes al ingreso ponían un cartel que decía «No tenemos Wi-Fi hablen entre ustedes», desde entonces nuevos términos se crearon como phubbing, que es la unión de las palabras phone and snubbing (teléfono e ignorar), es decir, el acto de ignorar el teléfono por un tiempo para conectarse con su grupo social y su entorno, este término a decir de los autores fue introducido en Estados Unidos el año 2007. Existe también un nuevo termino relacionado que es la nomofobia, que surge de no movile phone phobia, referido al miedo por no estar conectado al celular (Gutiérrez, 2015, como se citó en Escalada y Romero, 2015). 
Como una eficaz manera de contrarrestar la cada vez más creciente nomofobia, se propone la creación de espacios para recobrar la salud con programas enfocados a liberar a la persona de adicciones tecnológicas; a cargo de psicólogos y especialistas en terapias alternativas.

El objetivo de los tratamientos de bienestar es desconectar a la persona de la tecnología y reconectarla con su entorno, la naturaleza, las personas, la vida en general. Es una tendencia importante y necesaria en un futuro próximo, puesto que es cada vez más solicitada. Un estudio que refuerza esta teoría es el propuesto por el profesor Philip Pearce de la Universidad James Cook de Australia (como se citó en Lewis, 2018); él señala que los resort digitales de agujeros negros son opciones populares en las vacaciones de lujo en el Reino Unido y América del Norte y que la desintoxicación digital es una tendencia en aumento, que vale la pena observar para adaptarla a la oferta hotelera, así como programas acordes para responder a una creciente necesidad del mercado.

Las vacaciones digital detox, a decir del profesor Pearce, se hacen mediáticas a partir de 2011; sin embargo, es a partir del 2016 y 2017 que las vacaciones digitales se hacen más comunes y pasan de ser un nicho de mercado a ser una base amplia de consumidores (como se citó en Lewis, 2018).

En el contexto peruano, este tipo de programas podrían sumarse como beneficio adicional en una empresa de alojamiento. Incluso, la ausencia de cobertura de Internet puede asumirse como un valor agregado al establecimiento ya que en el Perú existen alojamientos que ofrecen una experiencia de contacto con la naturaleza y las sociedades del entorno. Esta tendencia se puede aprovechar muy bien, como ejemplo de ello, tenemos a las empresas de ecoalojamiento que se encuentran en áreas naturales protegidas, donde el atractivo de la naturaleza y la expedición se combina perfectamente con la ausencia de Internet, llevando al turista a una conexión real con el entorno. 


\section{Conclusiones}

El objetivo de este estudio es conocer de forma certera y fundamentada las características de las personas que se desplazan por turismo de salud, consumiendo servicios de bienestar o wellness; estos viajeros buscan experiencias en salud integral que les permitan encontrar equilibrio emocional. Es un segmento que va en aumento (PROMPERÚ, 2018).

IPSOS, en el estudio titulado Affluent Intelligence, se refiere a 24500 viajeros estadounidenses y los nuevos turistas de experiencias. Identifican seis tipos de turistas de experiencias, y uno de ellos es el turista de experiencias wellness; que se estima es un segmento representado por más de 10 millones de personas que puede gastar más de 9000 dólares anuales en viajes (PROMPERÚ, 2018).

Las sociedades, cada vez más tecnificadas, albergan a personas que deben enfrentar circunstancias difíciles -como la que enfrenta el mundo en 2020 con la pandemia por COVID-19-, que generan estrés y desajustes en la salud; a lo que se suma la valoración de la belleza como una característica de lo saludable. Aspectos que inciden en que el interés por el turismo de bienestar o wellness se desarrolle con mayor fuerza.

Los efectos de la pandemia con el aislamiento social, la adaptación a los cambios, a la denominada nueva normalidad, aún con la incertidumbre sobre vacunas y contagios generan estados de estrés, ansiedad y otros trastornos emocionales haciendo que se valore más que nunca la posibilidad de salir y experimentar contacto con la naturaleza y vivir experiencias diferentes a la rutina diaria.

Los hoteles, por lo tanto, en esta nueva etapa tienen en el mercado de turistas de bienestar una oportunidad de desarrollo y crecimiento al ofrecer productos que este segmento de mercado valora y prefiere; aprovechando su ubicación en la naturaleza y uso de la medicina tradicional del lugar. Estas experiencias logran que el turista vea su viaje mucho más enriquecedor, pues no solo apreciará un destino nuevo, sino que se conectará con la naturaleza, cultura y técnicas ancestrales del destino anfitrión. 
Los factores turismo, naturaleza y salud están y estarán cada vez más ligados entre sí, las tendencias lo describen y sustentan, es el reto para los destinos y las empresas saber articular estos en favor de crear productos que satisfagan la nueva e indivisible necesidad de los viajeros.

Por lo tanto, es preciso seguir en la búsqueda de estrategias para el desarrollo del turismo de bienestar; realizar estudios de mercado dirigido a los viajeros que prefieren este tipo de turismo; conocer las características sociodemográficas: edad, sexo, nivel de instrucción, lugar de origen, etc.; tener registro de las tendencias de viaje de este segmento de mercado, en redes, preferencias de destino, servicios contratados, monto de gasto o inversión en el viaje, tiempo de permanencia y otros. Con respecto a la tendencia digital detox, es conveniente ampliar los estudios sobre sus beneficios y técnicas de aplicación como terapias de relajación y psicológicas. Al ser una tendencia creciente puede llegar a ser un producto muy cotizado en el rubro de los negocios de alojamiento.

\section{Figura 3}

Interacción y complemento de factores

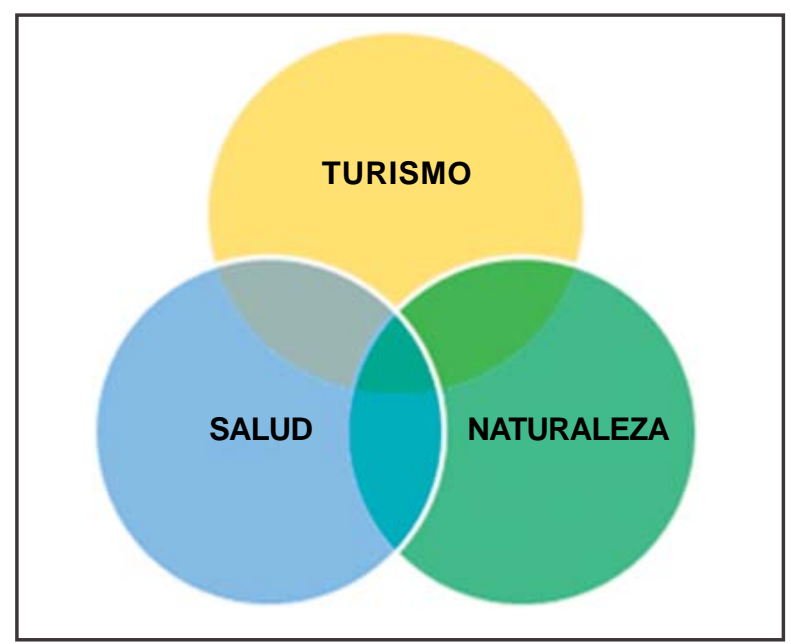

Nota. Elaboración propia. 


\section{Referencias}

Alberca, F. (2019). El turismo creativo. Conceptualización y características. Revista Cultura, 33, 145-160.

Arias, J., Caraballo, A. y Matos, R. (2012). El turismo de salud: conceptualización, historia, desarrollo y estado actual del mercado global. Clio America, 6(11), 72-98.

Comisión de Promoción del Perú para la Exportación y el Turismo (PROMPERÚ). (2018). Los nuevos turistas de experiencias. Turismo in, investiga innova, 49. https://www.promperu.gob.pe/turismoin/Boletines/2018/ sep/los_nuevos_turistas_de_experiencias.html

Comisión de Promoción del Perú para la Exportación y el Turismo (PROMPERÚ). (2019). Perfil del vacacionista nacional 2018.

Deloitte. (2018). Turismo Medico y Turismo de Bienestar. Más allá de una sala de espera. https:/l www2.deloitte.com/content/dam/Deloitte/mx/Documents/bienes-raices/2018/Turismo-medico-y-debienestar-2018.PDF

Escalada, K. y Romero, N. (2015). Estudio de Factibilidad de un Digital \& Body Detox Hotel (Tesis de titulación, Universidad Católica de Santiago de Guayaquil).

Escuela de Organización Industrial (EOI). (2013). Turismo de Salud en España. https://www.eoi.es/es/savia/ publicaciones/20788/turismo-de-salud-en-espana

Lewis, S. (2018, 24 de julio). Los viajeros se desconectan para unas vacaciones de desintoxicación digital. Maximpact.com News. http://www.maximpact.com/travelers-disconnect-digital-detox-holidays/

Ministerio de Comercio Exterior y Turismo (MINCETUR). (2012, diciembre). Estrategia de turismo termal en Perú. Informe final (T\&L Europraxis). https://www.mincetur.gob.pe/wp-content/uploads/documentos/turismo/ consultorias/estudiosConsultorias/PeruEstrategiaTurismoTermal-InformeFinal.pdf

Organización Mundial del Turismo (OMT). (2018). Panorama OMT del turismo internacional. https://www.eunwto.org/doi/pdf/10.18111/9789284419890

Organización Mundial del Turismo (OMT). (2019). Definiciones de turismo de la OMT. https://doi.org/10.18111/ 9789284420858

Oxford English Dictionary. (2020). https://www.oed.com/

Paunero, X. (2004). Patrimonio histórico y turismo cultural, un dialogo necesario en el desarrollo local de espacios periféricos. Revista Turismo y Patrimonio, 4, 89-101.

Rojas, M. (2018). El turismo termomedicinal en los baños termales de la Collpa y su influencia en el desarrollo turístico de la provincia de Huaral - Región Lima (Tesis doctoral, Universidad de San Martin de Porres), Lima, Perú.

Sánchez, S. (2018). Tendencias, perfiles y motivaciones del turismo de salud y bienestar. The Ostelea Tourism Management School. http://www.aept.org/archivos/documentos/informe_turismo_de_salud_y_bienestar _ostelea.pdf

Urbano, H. (2000). Patrimonio y Modernidad. Revista Turismo y Patrimonio, 1, 13-25.

Urry, J. (2004). La mirada del turista. Universidad de San Martín de Porres. 